\title{
Effect of Contralateral Prophylactic Mastectomy on Overall Survival in Patients with Hormone Receptor Negative Breast Cancer
}

Yong Cheng Su ( $\nabla$ syczb2643937537@163.com )

The First Affiliated Hospital of Xiamen University

XiaoGang Zheng

The First Affiliated Hospital of Xiamen University

\section{Research}

Keywords:

Posted Date: July 7th, 2021

DOl: https://doi.org/10.21203/rs.3.rs-674088/v1

License: (c) (1) This work is licensed under a Creative Commons Attribution 4.0 International License.

Read Full License 


\section{Abstract}

Introduction: There has been little research on whether contralateral prophylactic mastectomy(CPM) improves overall survival in patients with hormone receptor(HR) negative breast cancer. Our study aimed to determine whether CPM would bring survival benefits for the patients diagnosed with HR-negative breast cancer.

Methods: All data were derived from the SEER database (version8.3.6.1). We used LASSO regression analysis and stepwise regression method to screen the variables related to prognosis. The survival curves of breast cancer patients were drawn by the Kaplan-Meier method, and we used the Log-rank test to calculate statistical differences in survival curves.

Results: The overall survival (OS) was significantly better for patients in the CPM group than the NonCPM group, the pooled hazard ratio (HR) was 0.46 (95\% Cl: 0.14-0.78), multivariate Cox regression analysis after propensity score matching (PSM) showed the same results, the CPM-group showed a significantly improved prognosis than the Non-CPM group, the pooled HR was 0.66 (95\% Cl:0.50-0.83), statistically significant.

Conclusion: Our study confirmed that CPM could improve the prognosis of patients with HR-negative breast cancer and significantly extend overall survival. Simultaneously, clinicians should fully consider the advantages and disadvantages of the operation before performing CPM on patients.

\section{Background}

Breast malignancy is the most prevalent cancer among women in the world today[1, 2], and it is also cancer leading to the largest number of cancer-related mortality worldwide[3]. Based on reliable epidemiological evidence, more than 1 million new breast cancer cases were diagnosed in only 2012[4], and it was the second popular cancer, following only lung carcinoma [5]. Recent data show that breast carcinoma has replaced lung cancer with the highest incidence of malignant tumors globally. In 2018, more than 2 million newly diagnosed breast malignancies in the US accounted for about one-third of all new malignancies in American women[6],resulting in approximately 40000 fatalities[7].

Studies have pointed out that for patients diagnosed with unilateral breast cancer(UBC), the risk of contralateral breast cancer(CBC) is estimated to be 3-4 times higher than the general population[8, 9]. A retrospective study based on the SEER database showed that the 10-year and 20-year incidence of CBC was $6.1 \%, 12 \%$, respectively, for patients with UBC[10]. Therefore, some physicians performed contralateral prophylactic mastectomy (CPM) to reduce the incidence of $\mathrm{CBC}$. Furthermore, the data showed that the use of CPM has increased over time[11, 12]. Although doctors can prevent contralateral breast cancer by performing CPM, it is worth considering whether CPM will bring survival benefit for the patient; what's worse, due to operative trauma and complications, the surgeon needs to carefully weigh the necessity for CPM. There has been little research on whether CPM improves overall survival in 
patients with HR-negative breast cancer. Our study aimed to determine whether CPM would bring survival benefit for the patients diagnosed with HR negative breast cancer.

\section{Materials And Methods. \\ 2.1 Data collection}

Patients diagnosed with HR-negative stage I-III postoperative breast cancer were included in our research. All data were derived from the SEER database(version8.3.6.1), Collected the following data: age, sex, race, marital status, tumor size, tumor site, grade, TNM stage, estrogen receptor(ER) status, progesterone receptor(PR) status, HER2 status, molecular subtypes, surgery, chemotherapy record and radiotherapy record, and the primary endpoint was overall survival (OS).Continuous variable age was transformed into categorical variable using X-TILE software(version 3.6.1)[13, 14].

\subsection{Subjects}

Patients meeting the following criteria were included: patients who histologically confirmed invasive ductal carcinoma of the breast, HR(ER/PR)-negative, stage I-III, no distant metastasis, undergone surgery, one primary carcinoma only, and complete survival data were available.

\subsection{Statistical analysis:}

We used LASSO regression analysis and stepwise regression method to screen the variables related to prognosis, and then we performed multivariate Cox analyses to analyze the prognostic variables. The survival curves of breast cancer patients were drawn by the Kaplan-Meier method, and we used the Logrank test to calculate statistical differences of survival curves. The differences between categorical variables were compared using the chi-square test. The Propensity Score Matching (PSM) method was utilized to eliminate the effect of the confounding factors[15]. We matched the patients in the CPM group 1:1 with the patients in the non-CPM group[16]. Statistical analysis was conducted by using $R$ software(version 4.0.2), P-value $<0.05$ was considered statistically significant[17].

\section{Results}

\subsection{Baseline characteristics of included patients}

A total of 5739 female patients with HR-negative breast tumor were enrolled in our research. 2047 (35.7\%) patients who received a contralateral prophylactic mastectomy(CPM) were in the CPM group, and the other 3692 (64.3\%) patients were in the Non-CPM group. All included patients, baseline characteristics are shown in Table 1. 76.3\% of patients were younger than 65 years, the histological grade of enrolled patients $(76.3 \%)$ was grade III, Most of the patients had received chemotherapy $(79.0 \%)$ or radiotherapy (33.7\%). There were 3920 (68.3\%) patients with triple-negative breast cancer (TNBC), and 1819 patients were HER2-enriched breast cancer. According to the 6th AJCC staging system, 2634(45.9\%) patients were diagnosed with T2 and 3196 (55.7)\%) patients with N1 stage. 


\subsection{LASSO regression screened overall-survival-related variables}

As shown in Fig. 1, of the variables collected from eligible patients, 8 variables were selected using LASSO regression analysis (Fig. 1.B). There were age, grade, radiation recode, and chemotherapy recode, Stage, T stage, $\mathrm{N}$ stage and HER2 status.

\subsection{Survival benefit by CPM}

Kaplan-Meier curve demonstrated that the prognosis was a significant difference between the CPM group and the Non-CPM group. The overall survival was significantly better for patients in the CPM group than the Non-CPM group ( $P=0.00$, Fig. 2.A); the pooled hazard ratio was 0.46 ( $95 \% \mathrm{Cl}$ : $0.14-0.78)$, statistically significant. As shown in table 2, there was no balance between the CPM-group and the Non-CPM group in clinicopathological features, so we did a propensity score matching (PSM) to rule out the effects of confounding factors. Finally, there were 2047 matched pairs after 1:1 matching, no statistically significant differences were found among the CPM-group and Non-CPM group in the matched cohort (table.2 ). Multivariate Cox regression analysis in the matched cohort showed the same results; in the survival curve, the CPM-group showed a significantly improved prognosis than the Non-CPM group. The pooled HR was 0.66(95\% Cl:0.50-0.83), statistically significant(Fig. 2B).

\subsection{Survival benefit of CPM-group by subtype and other factors}

As shown in Table 2, CPM, together with age, grade, radiation recode, chemotherapy recode, Stage, $T$ stage, $\mathrm{N}$ stage, and HER2 status, were significantly independent prognostic predictors in the matched cohort. We performed subgroup analysis according to age, grade, radiation recode, chemotherapy recode, Stage, T stage, $\mathrm{N}$ stage, and HER2 status to identify patients who might benefit from CPM.

In CPM-group, triple-negative breast cancer(TNBC) patients had a significant reduction in mortality, and the pooled HR was 0.67 (95\% Cl:0.51-0.83, Fig. 3A), statistic important. Likewise, the same result was obtained with the HER2-enriched breast cancer ( $\mathrm{HR}=0.55,95 \% \mathrm{Cl}: 0.31-0.79, \mathrm{p}=0.012$, Fig. 3B). What s more, CPM bore prognostic significance among those patients who were younger than 65 years old, grade III, stage IIA, and IIIA (all P<0.05, Fig. 4). HR-negative breast cancer patients who received CPM with or without chemotherapy/radiotherapy would also observe survival benefits (all $P<0.05$, Fig. 5 and Fig. 6). All the Hazard ratios and $95 \%$ confidence interval for overall survival stratified by subgroup analysis were shown in Fig. 7.

\section{Discussion}

Consistently, CPM is a hotly debated surgery; there is no clear consensus on whether CPM improves HRnegative breast cancer patients[18-20]. Bedrosian and his colleagues pointed out that CPM could improve the prognosis of breast cancer patients, especially in young women with ER-negative early-stage 
breast cancer. Compared with the non-CPM group, they observed that patients who received CPM had a better prognosis; the pooled HR was $0.68(95 \% \mathrm{Cl}: 0.53-0.88, \mathrm{P}=0.004)$ in his study[20]. Their conclusion was the same as our research. In our study, CPM was an independent prognostic factor in HR-negative breast cancer patients. Multivariate Cox regression analysis showed that the HR of CPM-group v.s nonCPM group was $0.46(95 \% \mathrm{Cl}: 0.14-0.78)$, approximately a $54 \%$ reduction in the mortality rate was observed with patients who received CPM. Moreover, PSM was performed to eliminate the influence of differences among covariates on our results; after PSM, we found that our products remained consistent. The pooled HR was $0.66(95 \% \mathrm{Cl}: 0.56-0.80, \mathrm{P}<0.0001)$, which showed that our conclusions were robust and reliable.

Katja Goldflam's study pointed out that whether CPM had a survival benefit depended on several factors. In his research, breast cancer patients with invasive lobular carcinoma, hormone receptor (HR) positive, high-risk pathological classification, and older than 40 years of age might benefit from CPM[8]. Of course, the balance between the survival benefits of CPM and the adverse effects of CPM surgery itself should be carefully considered by each clinician and patient. Katja Goldflam's research showed that CPM was associated with a $90 \%$ reduction in contralateral breast cancer risk and a relative increase in surgical complications. Much worse, the delay in the adjuvant treatment of breast cancer caused by severe complications after CPM surgery can also adversely affect the outcome of patients. Simultaneously, CPM surgery will also increase the length of hospital stay and additional surgical costs, which is undoubtedly a significant burden for patients[21]. The study of Erin E Burke et al.[21] also pointed out that although CPM had many risks and complications, most patients were generally satisfied with CPM surgery. Their conclusion may be that CPM surgery alleviates patients' concerns about lateral breast cancer, the founding of Erin E Burke et al., which is consistent with the findings of Marlene H. Frost.

In the study of Marlene H. Frost, a total of 621 patients were followed up for more than ten years. The majority of patients (about 83\%) were satisfied with CPM; only a small number of patients were dissatisfied with the CPM for the complications, high cost, heavy burden, poor postoperative appearance[22]. Therefore, although most surgical patients are satisfied with CPM, CPM's adverse effects should be taken seriously and carefully weighed by every clinician and breast cancer patient. Considering the negative impact of CPM on patients' physiology and psychology and the risk of the operation itself, clinicians should fully consider the advantages and disadvantages of the process before performing CPM on patients to avoid patients' decision regret. As a result, the National Comprehensive Cancer Network (NCCN) guidelines only recommend that women with a genetic or familial high risk of breast cancer consider CPM an option for surgical procedures[23].

Any study has limitations, and ours is no exception. Firstly, our data comes from the SEER database, which is inevitably limited. The SEER database's data lacks a specific treatment plan and treatment cycle in patients[24]. The lack of such critical information is likely to reduce the effectiveness of our research[25], another is that these factors are closely related to the survival and prognosis of breast cancer patients(such as the presence of vascular tumor thromb infiltration, the waiting time between surgery and chemotherapy/radiotherapy, and whether the surgical margin is clean). The clinical 
characteristics are not available in the SEER database. Secondly, the HER2 receptor status in breast cancer patients was not collected in the SEER database until 2010, and our research mainly focused on breast cancer patients with well-defined molecular subtypes. Therefore, HR-negative breast tumor patients enrolled in the research of follow-up time are limited. Less information is gathered during the follow-up period, such as whether to accept patients with targeted therapy, leading to a decline in our reliability research. Thirdly, our study only considered the risk of death in patients with HR-negative breast tumor and did not consider the risk of recurrence or disease.

\section{Conclusion}

Our study confirmed that CPM surgery could improve the prognosis of HR-negative breast tumor patients and significantly extend overall survival. Considering the adverse effects of CPM on patients' physiology and psychology and the risk of the operation itself, clinicians should fully consider the advantages and disadvantages of the process before performing CPM on patients.

\section{Declarations}

Ethical approval and consent to participate : All procedures performed in studies involving human participants were in accordance with the ethical standards of the institutional research committee and with the 1964 Helsinki declaration and its later amendments or comparable ethical standards.

Consent for publication:Not applicable

Availability of data and materials:Not applicable

Informed consent :The study was approved by the local institutional review board, and the requirement for informed consent was waived because of the retrospective nature of this study.

Competing interests:The authors declare that they have no competing interests.

Funding :This research did not receive any specific grant from funding agencies in the public, commercial, or not-for-profit sectors.

Authors' contributions: S.Y .C and Z.X.G collated data and did analyses. S.Y.C planned and performed analyses,and was a major contributor in writing the manuscript. All authors read and approved the final manuscript.

Acknowledgements:Not applicable 


\section{References}

1. Tong Y, Wu J, Huang O, He J, Zhu L, Chen W, Li Y, Chen X, Shen K. 21-Gene Recurrence Score and Adjuvant Chemotherapy Decision for Breast Cancer Patients with Positive Lymph Nodes. SCI REPUK. 2019;9(1):13123.

2. Hu Y, Zhang J, Luo Q, Xu J, Yan Y, Mu L, Bai J, Lu W. Nanostructured Dihydroartemisinin Plus Epirubicin Liposomes Enhance Treatment Efficacy of Breast Cancer by Inducing Autophagy and Apoptosis. Nanomaterials (Basel Switzerland). 2018;8(10):804.

3. Raphael MJ, Biagi JJ, Kong W, Mates M, Booth CM, Mackillop WJ. The relationship between time to initiation of adjuvant chemotherapy and survival in breast cancer: a systematic review and metaanalysis. Breast Cancer Res Treat. 2016;160(1):17-28.

4. Goldhirsch A, Ingle JN, Gelber RD, Coates AS, Thürlimann B, Senn HJ. Thresholds for therapies: highlights of the St Gallen International Expert Consensus on the primary therapy of early breast cancer 2009. ANN ONCOL. 2009;20(8):1319-29.

5. Guo P, Pu T, Chen S, Qiu Y, Zhong X, Zheng H, Chen L, Bu H, Ye F. Breast cancers with EGFR and HER2 co-amplification favor distant metastasis and poor clinical outcome. ONCOL LETT. 2017;14(6):6562-70.

6. Wang C, Chang Y, Kuo Y, Lee K, Chen P, Cheung CHA, Chang C, Phan NN, Shen M, Hsu H. Mutation of the PTCH1 gene predicts recurrence of breast cancer. SCI REP-UK. 2019;9(1):16359.

7. Walens A, DiMarco AV, Lupo R, Kroger BR, Damrauer JS, Alvarez JV. CCL5 promotes breast cancer recurrence through macrophage recruitment in residual tumors. ELIFE. 2019;8:e43653.

8. Contralateral prophylactic mastectomy: Predictors of significant histologic findings. CANCER-AM CANCER SOC 2004.

9. Pan B, Xu Y, Zhou Y, Yao R, Wu H, Zhu Q, Wang C, Mao F, Lin Y, Shen S, et al: The prognostic comparison among unilateral, bilateral, synchronous bilateral, and metachronous bilateral breast cancer: A meta-analysis of studies from recent decade (2008-2018). CANCER MED-US 2019, 8(6):2908-2918.

10. Gao X, Fisher SG, Emami B. Risk of second primary cancer in the contralateral breast in women treated for early-stage breast cancer: A population-based study - ScienceDirect. International Journal of Radiation Oncology*Biology*Physics. 2003;56(4):1038-45.

11. Tuttle TM, Habermann EB, Grund EH, Morris TJ, Virnig BA. Increasing Use of Contralateral Prophylactic Mastectomy for Breast Cancer Patients: A Trend Toward More Aggressive Surgical Treatment. Breast Diseases. 2008;19(1):52.

12. Sutton EJ, Watson EJ, Gibbons G, Goldman DA, Moskowitz CS, Jochelson MS, Dershaw DD, Morris EA. Incidence of Internal Mammary Lymph Nodes with Silicone Breast Implants at MR Imaging after Oncoplastic Surgery. RADIOLOGY. 2015;277(2):381-7.

13. Storr SJ, Lee KW, Woolston CM, Safuan S, Green AR, Macmillan RD, Benhasouna A, Parr T, Ellis IO, Martin SG. Calpain system protein expression in basal-like and triple-negative invasive breast cancer. 
Annals of oncology: official journal of the European Society for Medical Oncology. 2012;23(9):228996.

14. Wu M, Li X, Zhang T, Liu Z, Zhao Y. Identification of a Nine-Gene Signature and Establishment of a Prognostic Nomogram Predicting Overall Survival of Pancreatic Cancer. FRONT ONCOL. 2019;9:996.

15. Wańha W, Mielczarek M, Gilis-Malinowska N, Roleder T, Milewski M, Ładziński S, Ciećwierz D, Gąsior P, Pawłowski T, Januszek R, et al. Safety and Efficacy of Embolic Protection Devices in Saphenous Vein Graft Interventions: A Propensity Score Analysis-Multicenter SVG PCI PROTECTA Study. J CLIN MED. 2020;9(4):1198.

16. Hung C, Hsieh Y, Li C, Huang J, Lin C, Wu T. Age and CHADS2 Score Predict the Effectiveness of Renin-Angiotensin System Blockers on Primary Prevention of Atrial Fibrillation. SCI REP-UK. 2015;5:11442.

17. Fu J, Zuber J, Martinez M, Shonts B, Obradovic A, Wang H, Lau S, Xia A, Waffarn EE, Frangaj K, et al. Human Intestinal Allografts Contain Functional Hematopoietic Stem and Progenitor Cells that Are Maintained by a Circulating Pool. CELL STEM CELL. 2019;24(2):227-39.

18. Chen $\mathrm{H}$, Wu K, Wang M, Wang F, Zhang M, Zhang P: A standard mastectomy should not be the only recommended breast surgical treatment for non-metastatic inflammatory breast cancer. A large population-based study in the Surveillance, Epidemiology, and End Results database 18. BREAST 2017, 35:48-54.

19. Kurian AW, Lichtensztajn DY, Keegan THM, Nelson DO, Clarke CA, Gomez SL. Use of and Mortality After Bilateral Mastectomy Compared With Other Surgical Treatments for Breast Cancer in California, 1998-2011. Journal of the American Medical Association 2014, 312(9):902-914.

20. Isabelle B, Chung-Yuan H, Chang GJ. Population-Based Study of Contralateral Prophylactic Mastectomy and Survival Outcomes of Breast Cancer Patients. Journal of the National Cancer Institute (6):1372-1373.

21. Burke EE, Portschy PR, Tuttle TM. Contralateral prophylactic mastectomy: are we overtreating patients? EXPERT REV ANTICANC. 2014;14(5):491-4.

22. Frost MH. Satisfaction After Contralateral Prophylactic Mastectomy: The Significance of Mastectomy Type, Reconstructive Complications, and Body Appearance. Journal of Clinical Oncology Official Journal of the American Society of Clinical Oncology. 2005;23(31):7849.

23. Daly MB, Pilarski R, Berry M, Buys SS, Farmer M, Friedman S, Garber JE, Kauff ND, Khan S, Klein C, et al: NCCN Guidelines Insights: Genetic/Familial High-Risk Assessment: Breast and Ovarian, Version 2.2017. J Natl Compr Canc Netw 2017, 15(1):9-20.

24. Liu P, Wang K, Jin J, Bi X, Sun P, Wang Y, Yang H, Li Z, Jiang W, Xia Y. Role of radiation therapy in primary breast diffuse large B-cell lymphoma in the Rituximab era: a SEER database analysis. CANCER MED-US. 2018;7(5):1845-51.

25. Mateo AM, Mazor AM, Obeid E, Sigurdson ER, DeMora L, Handorf EA, Daly JM, Aggon AA, Bleicher RJ. Time to surgery and the impact of delay on triple negative breast cancers and other phenotypes. J CLIN ONCOL 2018, 36(15). 


\section{Tables}

Table 1. The baseline characteristics of patients included in our study. 


\begin{tabular}{|c|c|c|c|}
\hline \multirow[b]{2}{*}{ Characteristics } & \multicolumn{3}{|c|}{ Before PSM (5739) } \\
\hline & CPM group (\%) & Non-CPM group(\%) & P-value \\
\hline Total & 2047(35.7) & 3692(67.3) & \\
\hline Age (\%) & & & $<0.001$ \\
\hline$>65$ & $165(8.1)$ & $1198(32.4)$ & \\
\hline$\leq 65$ & 1882(91.9) & $2494(67.6)$ & \\
\hline Grade (\%) & & & 0.002 \\
\hline I & $22(1.1)$ & $44(1.2)$ & \\
\hline$\|$ & $293(14.3)$ & $671(18.2)$ & \\
\hline III & $1717(83.9)$ & $2952(80.0)$ & \\
\hline IV & $15(0.7)$ & $25(0.7)$ & \\
\hline Radiation.recode $=$ Yes (\%) & $674(32.9)$ & $1258(34.1)$ & 0.394 \\
\hline Chemotherapy.recode $=$ Yes (\%) & $1788(87.3)$ & $2747(74.4)$ & $<0.001$ \\
\hline $\begin{array}{l}\text { Adjusted.AJCC.6th.Stage } \\
\text { (\%) }\end{array}$ & & & $<0.001$ \\
\hline I & $639(31.2)$ & $923(25.0)$ & \\
\hline$\| A$ & $663(32.4)$ & $1119(30.3)$ & \\
\hline IIB & $363(17.7)$ & $687(18.6)$ & \\
\hline IIIA & $252(12.3)$ & $503(13.6)$ & \\
\hline IIIB & 48 ( 2.3$)$ & $192(5.2)$ & \\
\hline IIIC & $82(4.0)$ & $268(7.3)$ & \\
\hline Adjusted.AJCC.6th.T (\%) & & & $<0.001$ \\
\hline T1a & $91(4.4)$ & $195(5.3)$ & \\
\hline T1b & 179 ( 8.7) & $239(6.5)$ & \\
\hline T1c & $537(26.2)$ & $745(20.2)$ & \\
\hline T1mic & $28(1.4)$ & $74(2.0)$ & \\
\hline $\mathrm{T} 2$ & $925(45.2)$ & 1709 (46.3) & \\
\hline T3 & $227(11.1)$ & $485(13.1)$ & \\
\hline $\mathrm{T} 4$ & 60 ( 2.9) & 245 (6.6) & \\
\hline
\end{tabular}




\begin{tabular}{|cccc|}
\hline Adjusted.AJCC.6th.N (\%) & & & $<0.001$ \\
N0 & $1215(59.4)$ & $1981(53.7)$ & \\
N1 & $592(28.9)$ & $1074(29.1)$ & \\
N2 & $158(7.7)$ & $369(10.0)$ & \\
N3 & $82(4.0)$ & $268(7.3)$ & \\
\hline HER2 = positive (\%) & $578(28.2)$ & $1241(33.6)$ & $<0.001$ \\
\hline
\end{tabular}

Table 2. The baseline characteristics of patients included in our study after PSM. 


\begin{tabular}{|c|c|c|c|}
\hline \multirow[b]{2}{*}{ Characteristics } & \multicolumn{3}{|c|}{ Before PSM (5739) } \\
\hline & CPM group (\%) & Non-CPM group(\%) & P-value \\
\hline Total & 2047(35.7) & 3692(67.3) & \\
\hline Age (\%) & & & $<0.001$ \\
\hline$>65$ & $165(8.1)$ & $1198(32.4)$ & \\
\hline$\leq 65$ & 1882(91.9) & $2494(67.6)$ & \\
\hline Grade (\%) & & & 0.002 \\
\hline 1 & $22(1.1)$ & $44(1.2)$ & \\
\hline$\|$ & $293(14.3)$ & $671(18.2)$ & \\
\hline III & $1717(83.9)$ & $2952(80.0)$ & \\
\hline IV & $15(0.7)$ & $25(0.7)$ & \\
\hline Radiation.recode $=$ Yes (\%) & $674(32.9)$ & $1258(34.1)$ & 0.394 \\
\hline Chemotherapy.recode $=$ Yes (\%) & $1788(87.3)$ & $2747(74.4)$ & $<0.001$ \\
\hline $\begin{array}{l}\text { Adjusted.AJCC.6th.Stage } \\
\text { (\%) }\end{array}$ & & & $<0.001$ \\
\hline 1 & 639 (31.2) & $923(25.0)$ & \\
\hline$\| A$ & $663(32.4)$ & $1119(30.3)$ & \\
\hline IIB & $363(17.7)$ & $687(18.6)$ & \\
\hline IIIA & $252(12.3)$ & $503(13.6)$ & \\
\hline IIIB & 48 ( 2.3$)$ & $192(5.2)$ & \\
\hline IIIC & $82(4.0)$ & $268(7.3)$ & \\
\hline Adjusted.AJCC.6th.T (\%) & & & $<0.001$ \\
\hline T1a & $91(4.4)$ & $195(5.3)$ & \\
\hline $\mathrm{T} 1 \mathrm{~b}$ & 179 ( 8.7) & $239(6.5)$ & \\
\hline T1c & $537(26.2)$ & $745(20.2)$ & \\
\hline T1mic & $28(1.4)$ & $74(2.0)$ & \\
\hline $\mathrm{T} 2$ & $925(45.2)$ & 1709 (46.3) & \\
\hline T3 & $227(11.1)$ & $485(13.1)$ & \\
\hline $\mathrm{T} 4$ & 60 ( 2.9) & 245 (6.6) & \\
\hline
\end{tabular}




\begin{tabular}{|llll|}
\hline Adjusted.AJCC.6th.N (\%) & & $<0.001$ \\
\hline N0 & $1215(59.4)$ & $1981(53.7)$ & \\
\hline N1 & $592(28.9)$ & $1074(29.1)$ & \\
\hline N2 & $158(7.7)$ & $369(10.0)$ & \\
\hline N3 & $82(4.0)$ & $268(7.3)$ & \\
\hline HER2 = positive (\%) & $578(28.2)$ & $1241(33.6)$ & $<0.001$ \\
\hline
\end{tabular}

Figures 
A

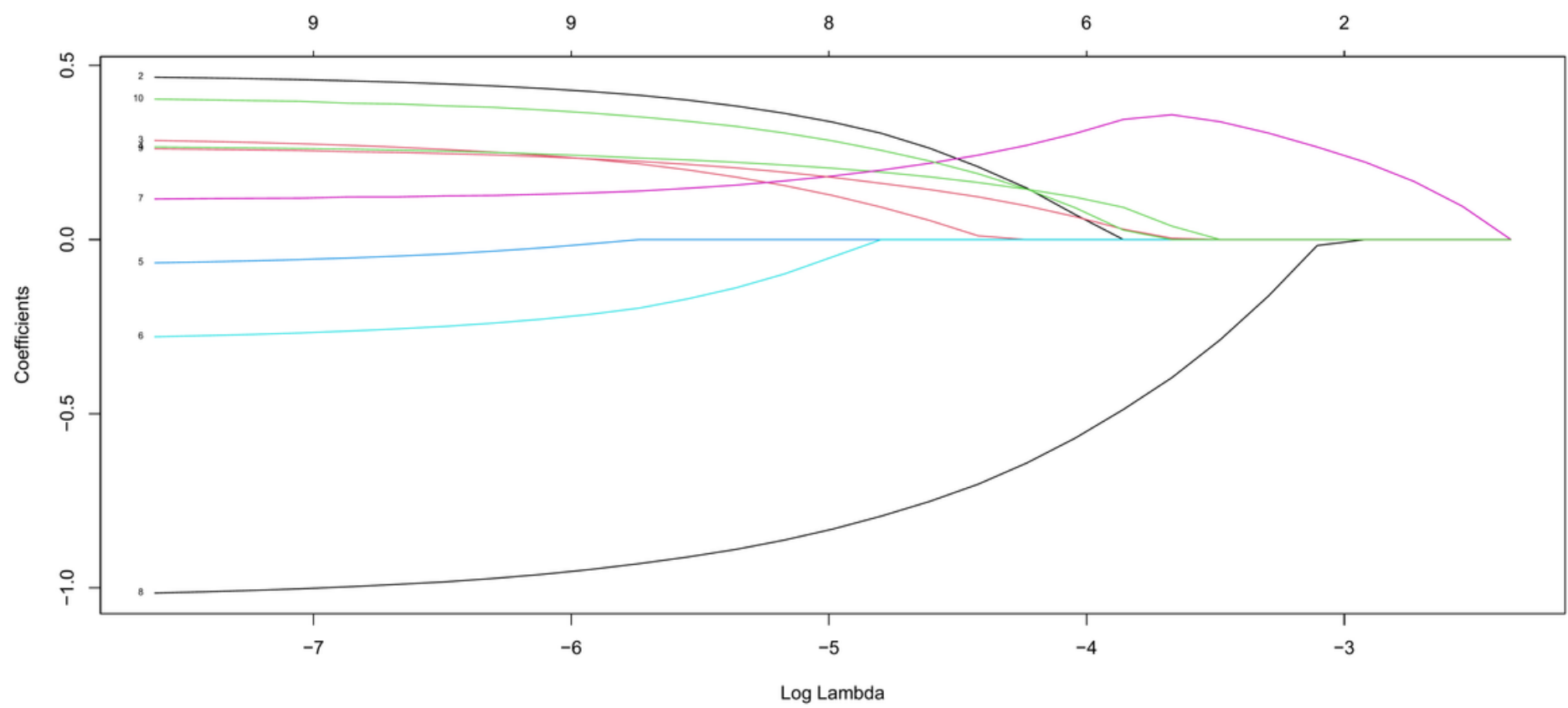

B



Figure 1

(A ) The LASSO plot, variables screened by the LASSO regression analysis, (B) the optimal value of $\lambda$ were selected by 10 cross-validations. 
A Overall survival

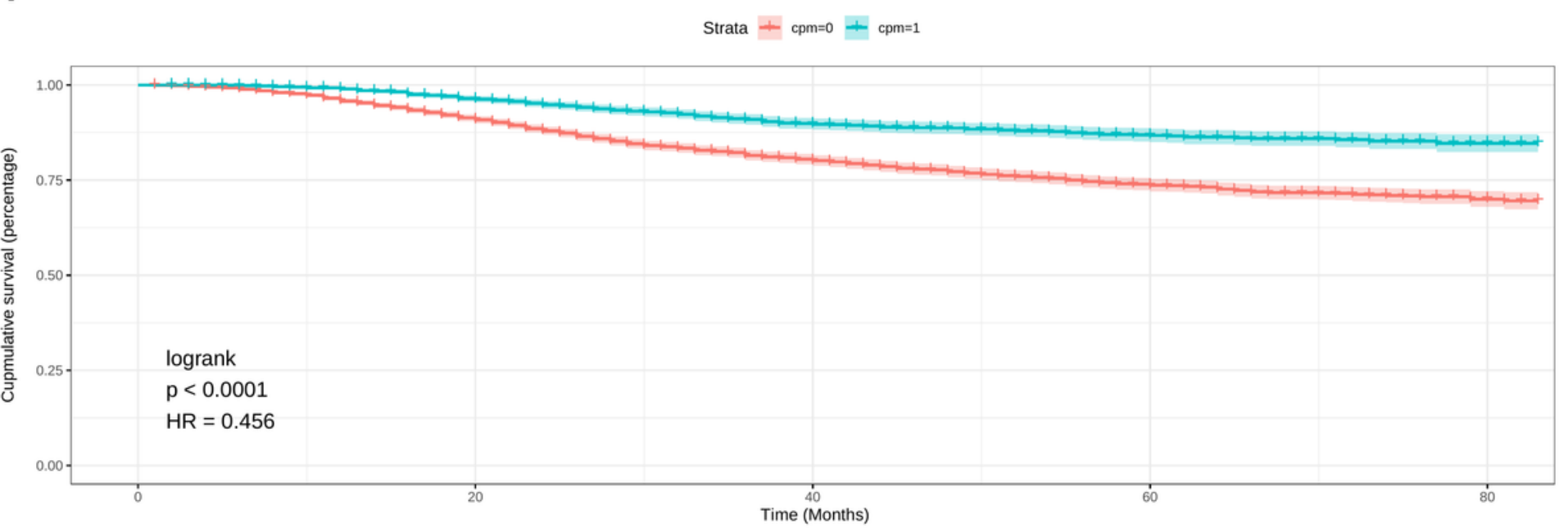

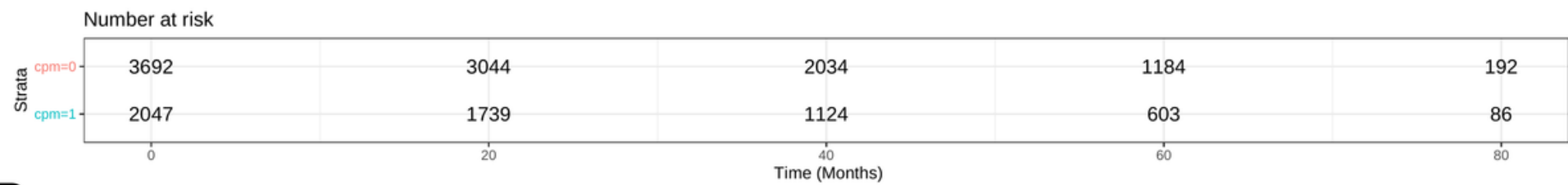

B Overall survival

Strata + cpm=0 $\div$ cpm=1

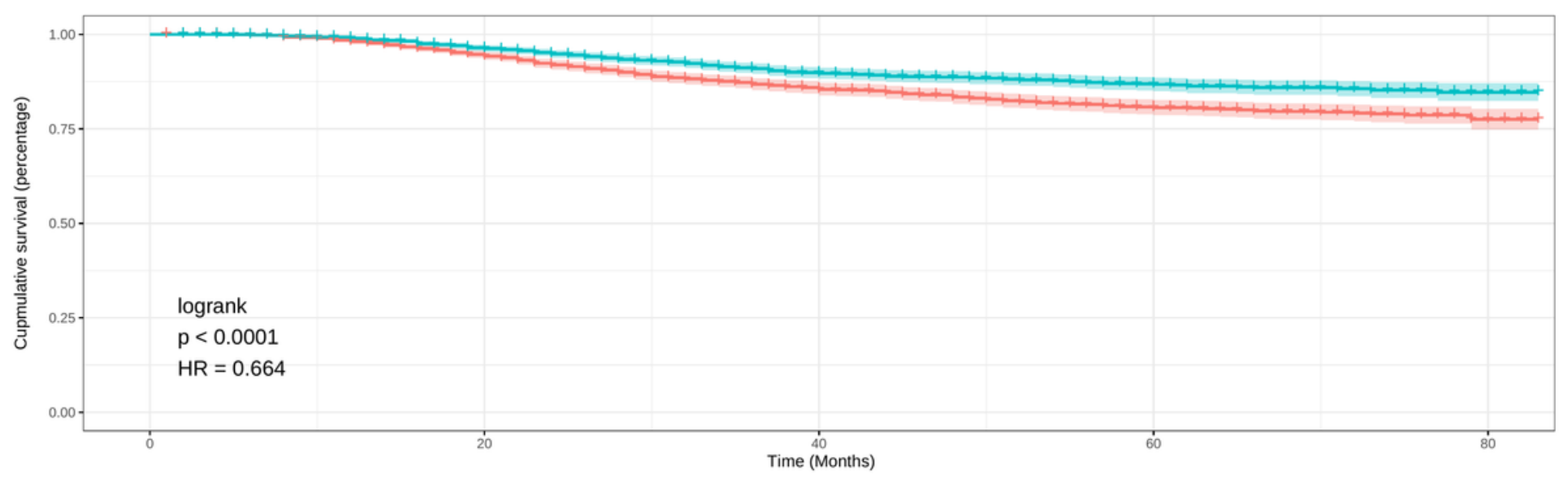

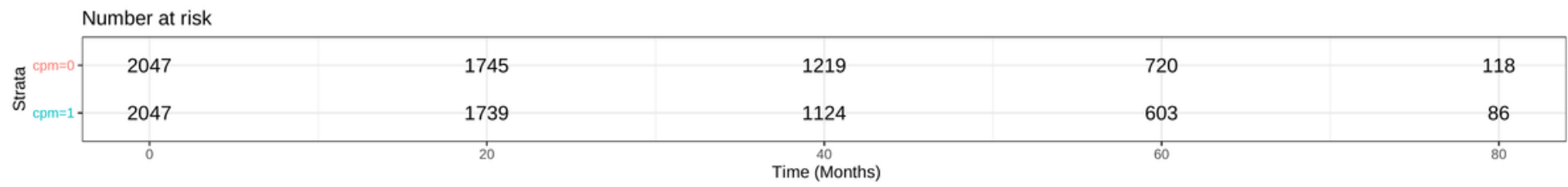

Figure 2

(A) Kaplan-Meier OS curves in the whole cohort before PSM. (B) Kaplan-Meier OS curves in the whole cohort after PSM. 
A Overall survival

Strata $+\mathrm{cpm}=0+\mathrm{cpm}=1$

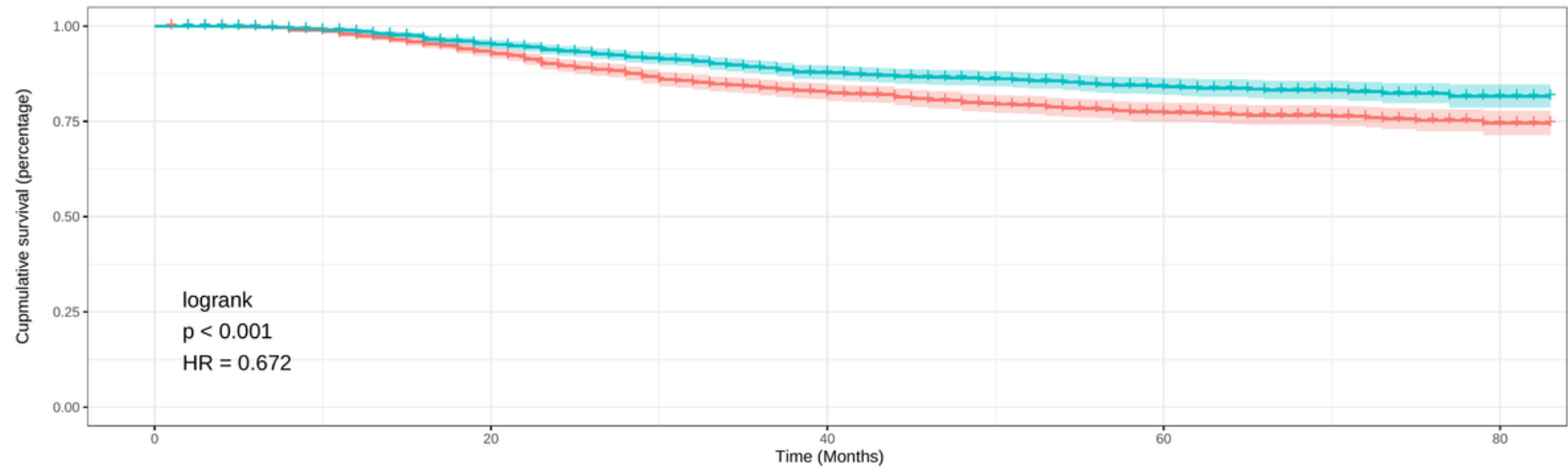

Number at risk

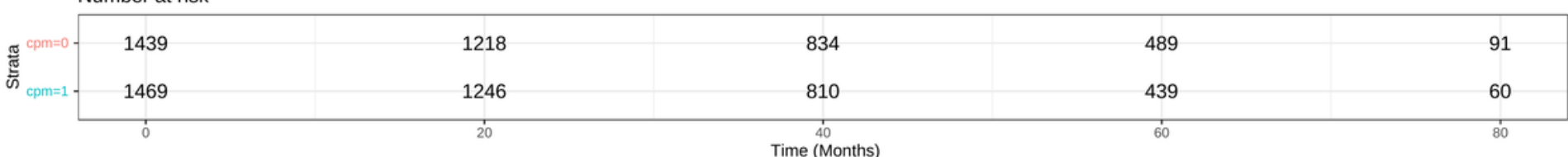

B Overall survival

Strata $+\mathrm{cpm}=0+\mathrm{cpm}=1$

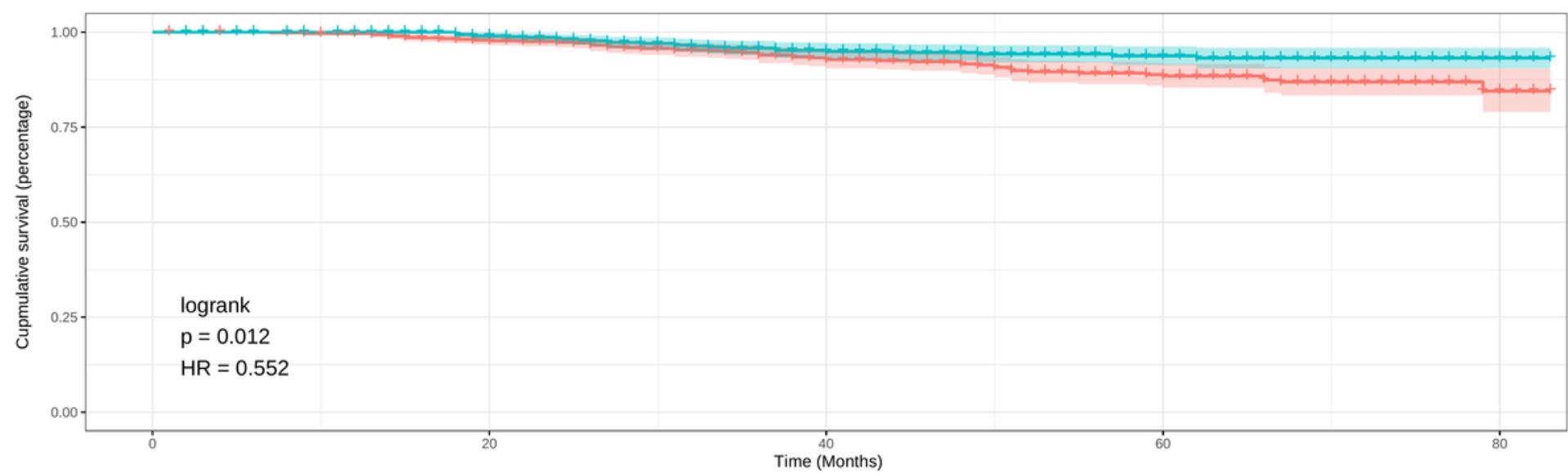

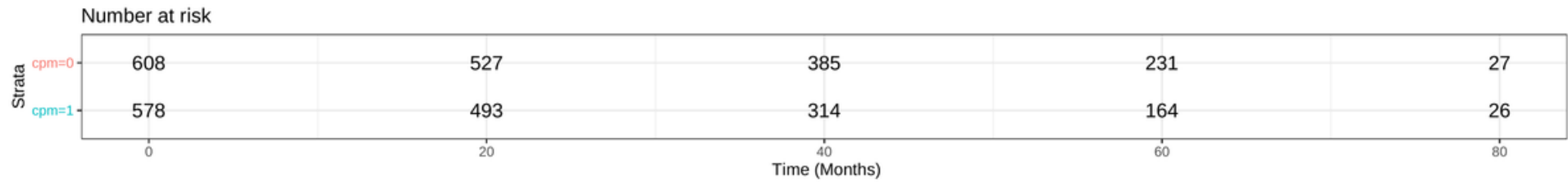

Figure 3

(A) Kaplan-Meier OS curves in the TNBC subtype.(B) HER2-enriched subtype. 

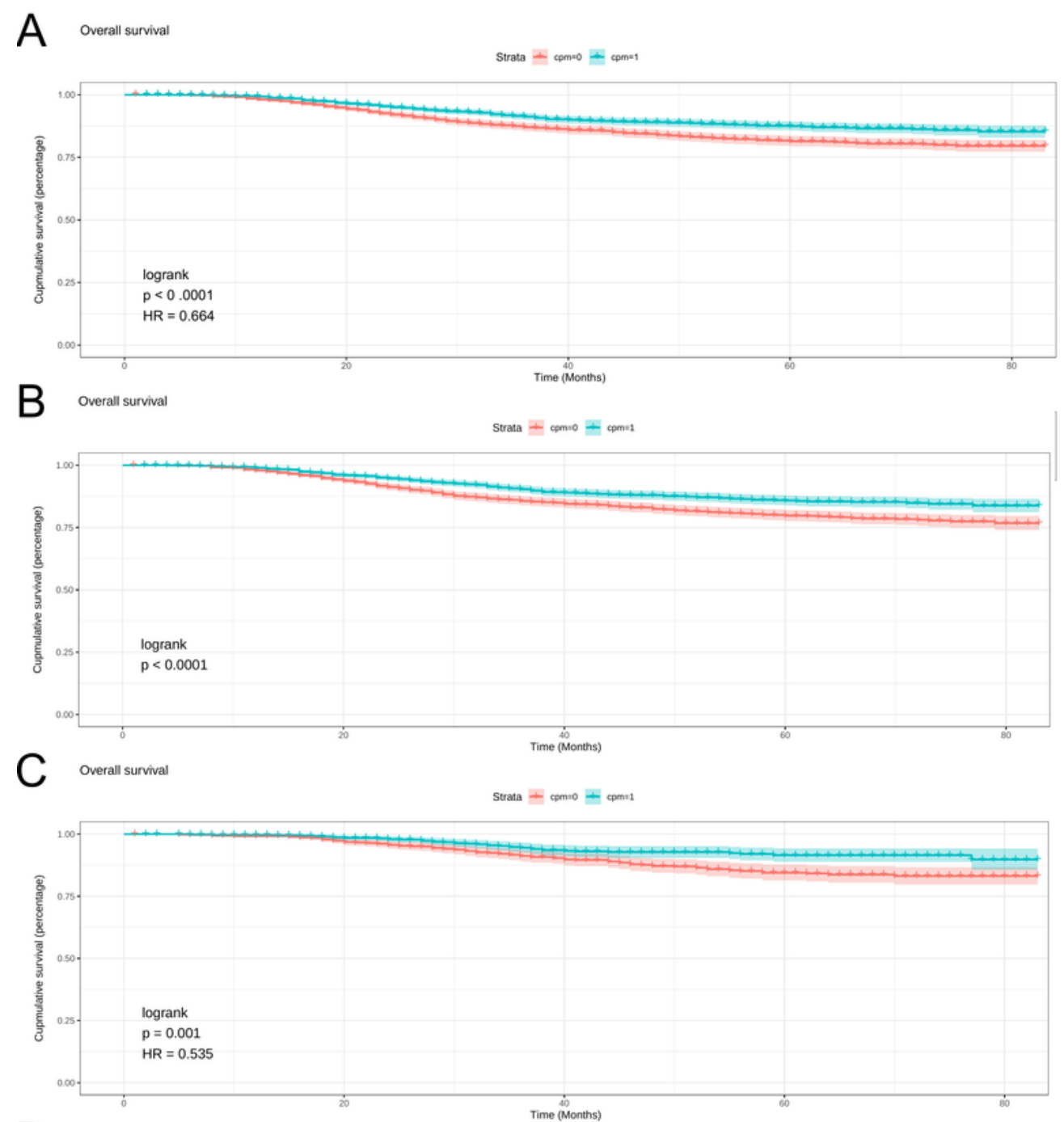

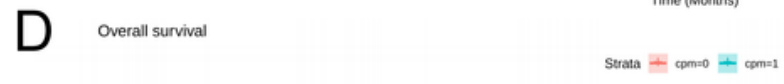

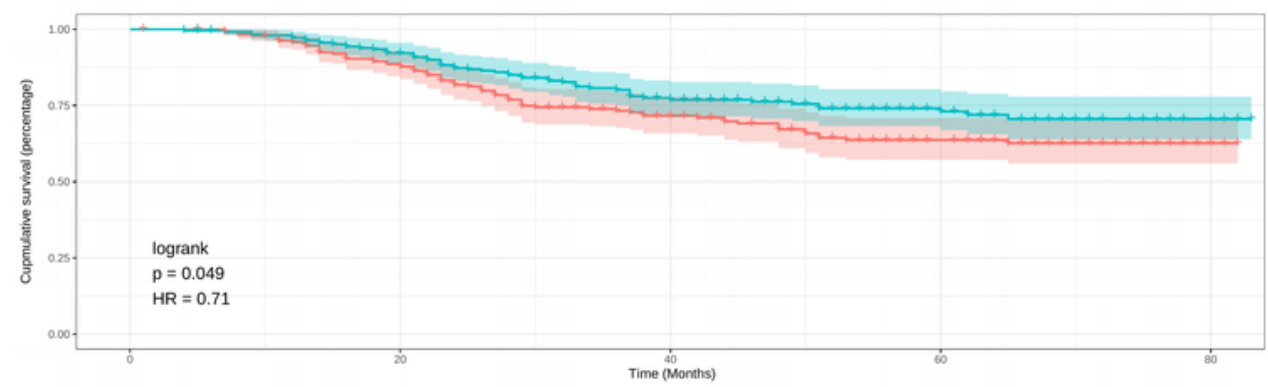

Figure 4

(A) Kaplan-Meier OS curves with younger than 65 years old,(B)grade III,(C) stage lla tumor,(D) stage Illa tumor. 
A Overall survival

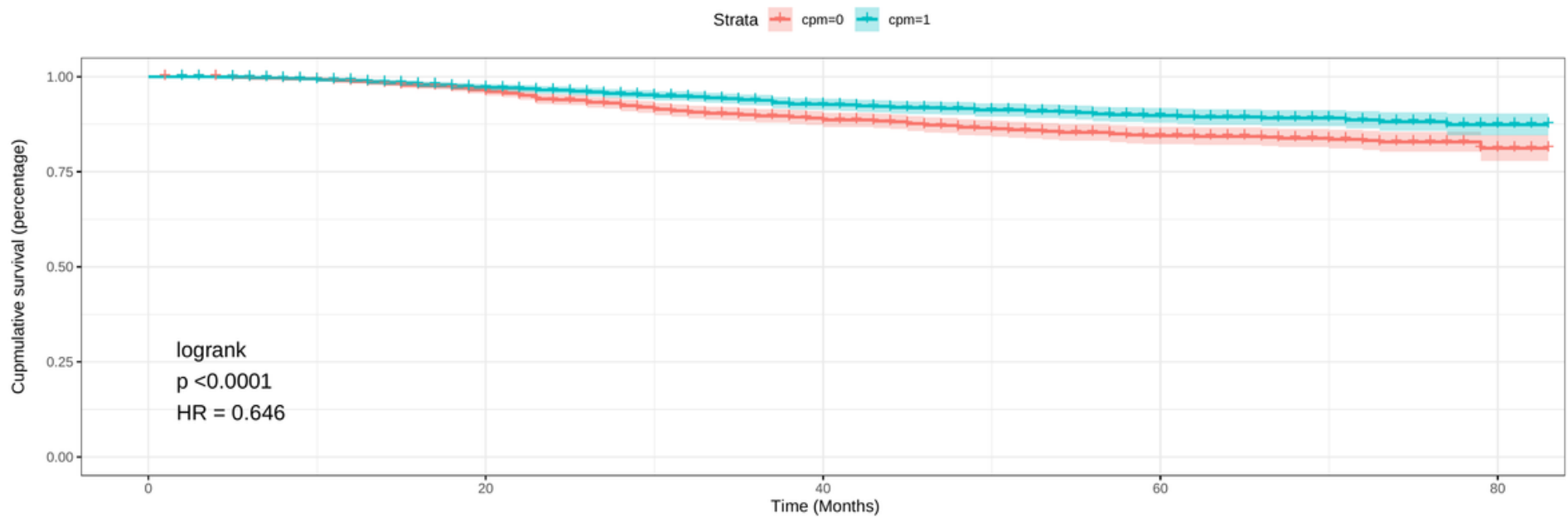

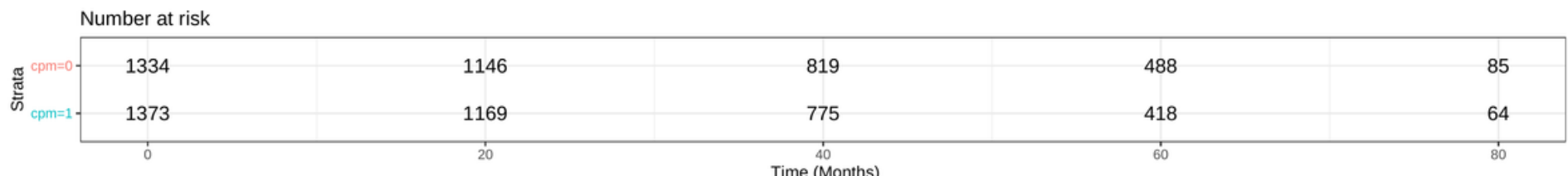
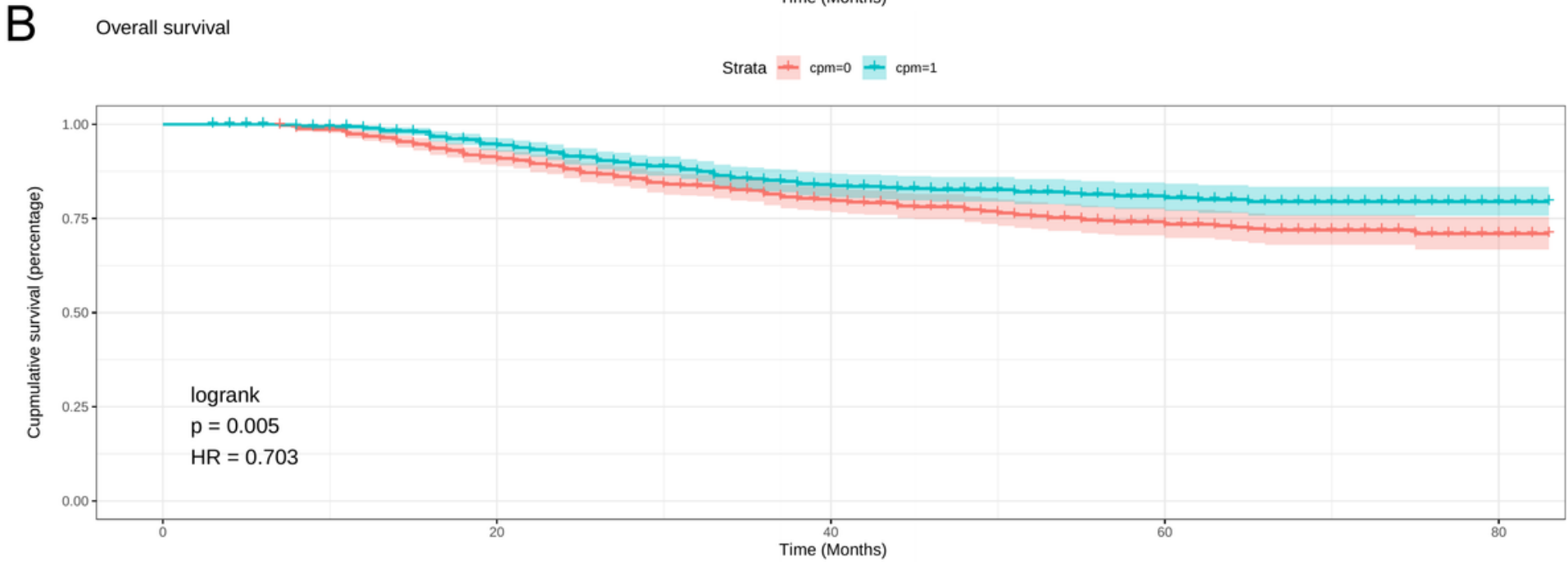

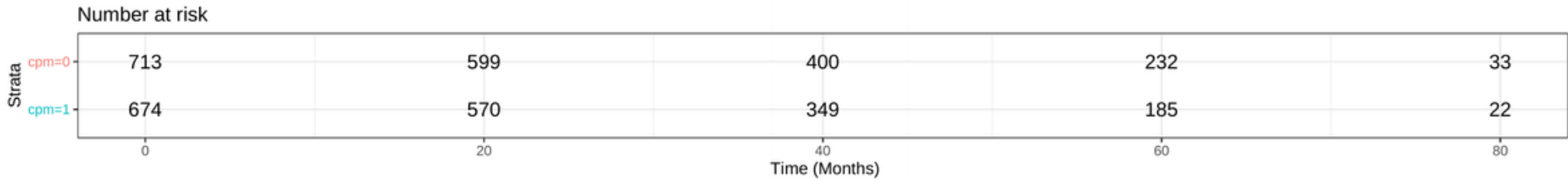

\section{Figure 5}

(A) Kaplan-Meier OS curves without radiotherapy,(B)with radiotherapy. 
A Overall survival

Strata $+{ }_{\text {cpm=0 }}+{ }_{\text {cpm=1 }}$

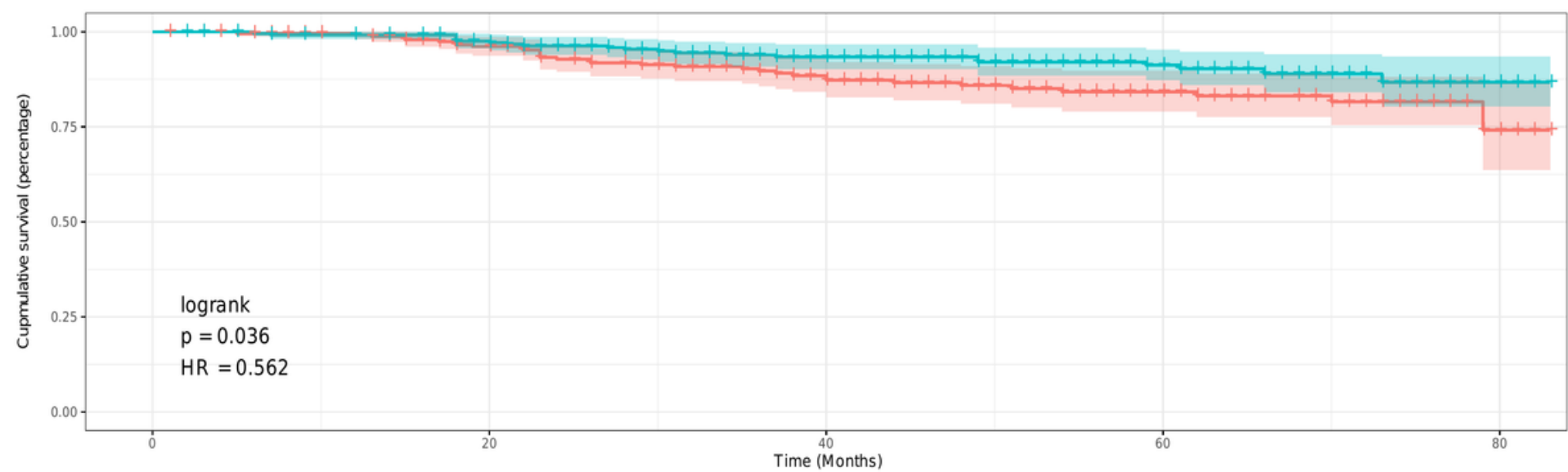

Number at risk

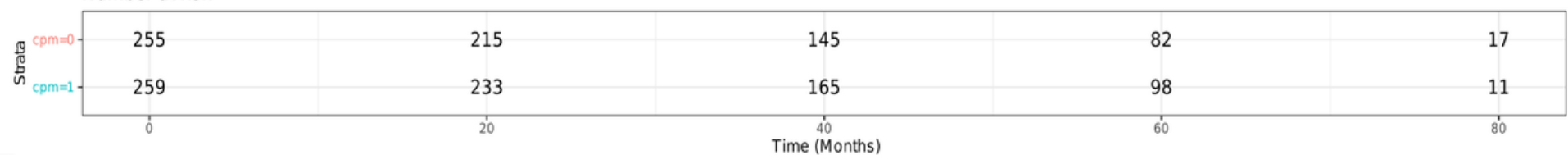

B Overall survival

Strata $+{ }_{\mathrm{cpm}=0}+\mathrm{cpm}_{\mathrm{c}} \mathrm{l}$

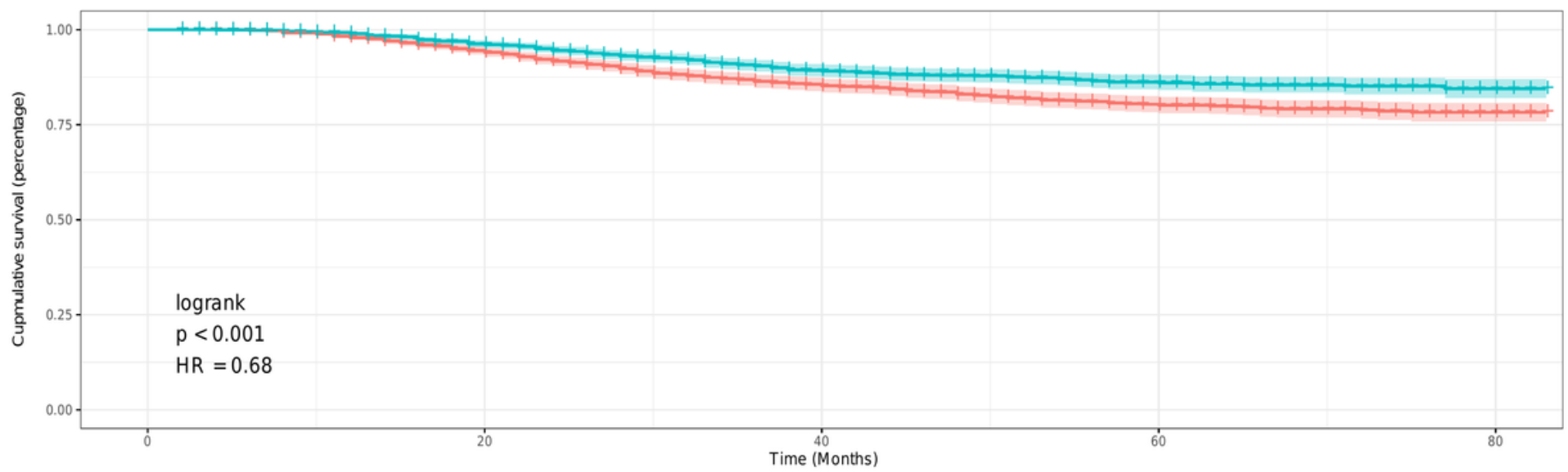

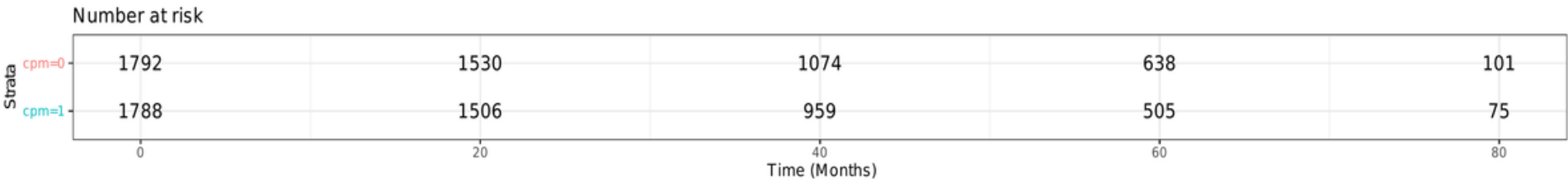

Figure 6

(A) Kaplan-Meier OS curves withiout chemotherapy,(B)with chemotherapy. 


\section{Overall Survival stratified by subgroup analysis}

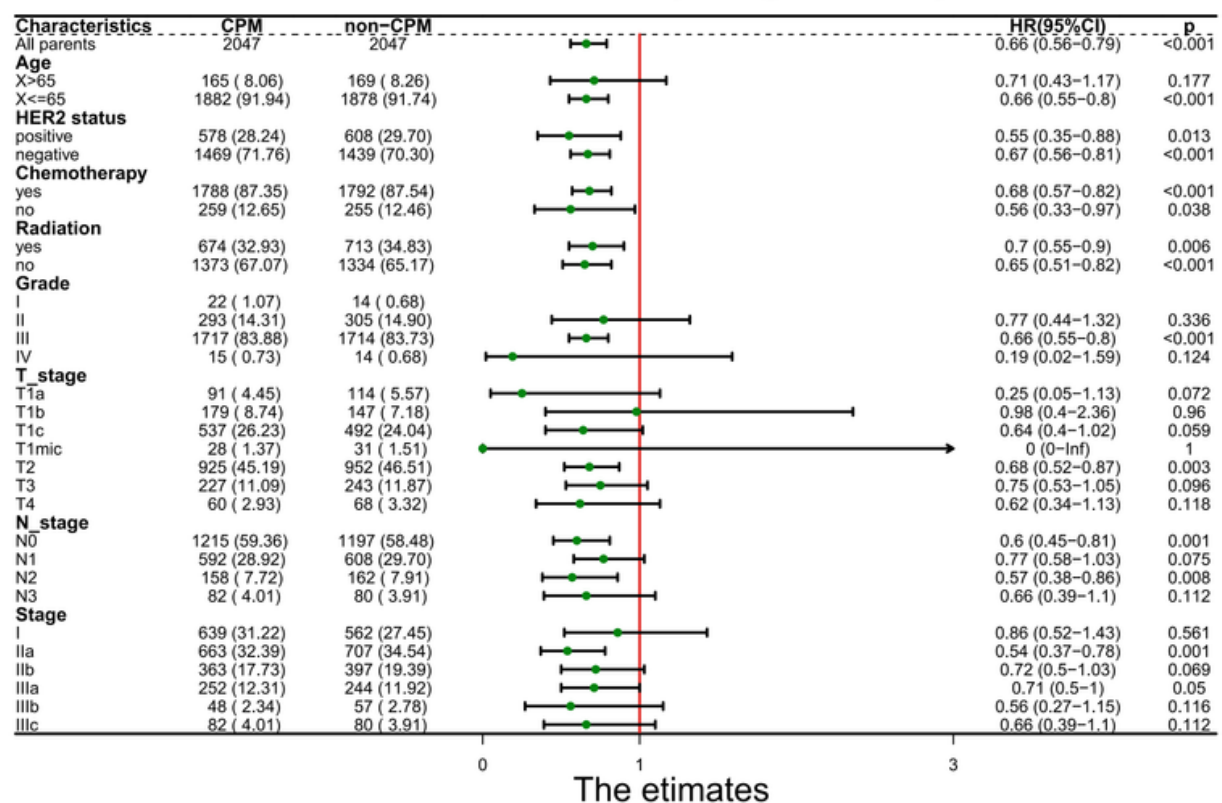

\section{Figure 7}

Hazard ratio and $95 \%$ confidence interval for overall survival stratified by subgroup analysis. 